\title{
Riscos de Acidente Vascular Encefálico como complicação neurológica em pacientes acometidos pela COVID-19
}

\author{
Risks of Stroke as a neurological complication in patients affected by COVID-19 \\ Riesgos de Ictus como complicación neurológica en pacientes afectados por COVID-19
}

Arquimedes Cavalcante Cardoso ORCID: https://orcid.org/0000-0001-9546-805X Universidade Federal do Piauí, Brasil E-mail: arquimedes@ufpi.edu.br

Milena Matos da Cruz

ORCID: https://orcid.org/0000-0002-9875-8906 Centro Universitátio ITPAC, Brasil E-mail: milenamc_5@hotmail.com

Vicente de Paula Freire da Silva Junior ORCID: https://orcid.org/0000-0002-2021-3818 Centro Universitátio ITPAC, Brasil

E-mail: vicentejunior96@ hotmail.com

Haroldo Bandeira de Matos Filho ORCID: https://orcid.org/0000-0003-0948-8017 Faculdade ITPAC, Brasi

E-mail: haroldohrpn@ @otmail.com

Karina Raminho Melo

ORCID: https://orcid.org/0000-0001-7094-2042

Universidade Federal do Pará, Brasil

E-mail: Karinaramonho95@gmail.com

Fabiane Jesus da Silva

ORCID: https://orcid.org/0000-0002-8604-3807

Centro Universitário Santo Agostinho, Brasil E-mail: fabhinie@hotmail.com

Elloá Martins Oliveira da Rocha

ORCID: https://orcid.org/0000-0001-7074-8426

Universidade Cidade de São Paulo, Brasil E-mail: elloamrocha@hotmail.com

Luciano Paes Landim Ribeiro

ORCID: https://orcid.org/0000-0002-5495-7938 Uninovafapi, Brasil

E-mail: lucym_srn@hotmail.com

Iaggo Henrique de Sousa Figueiredo ORCID: https://orcid.org/0000-0001-6136-0411

Universidade Estadual do Piauí, Brasil

E-mail: iaggo0106@hotmail.com

Jaqueline Dantas da Costa

ORCID: https://orcid.org/0000-0003-2570-1949

Faculdade Centro Universitário Uninovafapi, Brasil E-mail: jaquedc87@gmail.com

Joice Mara Ferreira Dos Santos

ORCID: https://orcid.org/0000-0002-3231-750X AESPI-Associação de Ensino Superior Do Piauí, Brasil E-mail: joicinha2.2kinha@gmail.com 


\author{
Geovana Marques Teixeira \\ ORCID: https://orcid.org/0000-0002-1182-8650 \\ Universidade Estadual do Piauí, Brasil \\ E-mail: geovanamarquest@gmail.com \\ Kaline Lousada Muniz \\ ORCID: https://orcid.org/0000-0002-3110-6946 \\ Centro Universitário INTA, Brasil \\ E-mail: enfermeirakalinemuniz@gmail.com \\ José Guilherme Férrer Pompeu \\ ORCID: https://orcid.org/0000-0001-9038-0833 \\ Universidade Federal do Piauí, Brasil \\ E-mail: gpompeu8@gmail.com \\ Mylena Silva da Silva \\ ORCID: https://orcid.org/0000-0002-4872-3131 \\ Universidade Federal do Piauí, Brasil \\ E-mail: mylenaa_silvaa@ hotmail.com
}

\begin{abstract}
Resumo
Tendo em vista o atual cenário pandêmico ocasionado pela COVID-19 pode gerar um significativo envolvimento cardiovascular, que é o principal fator de risco para o AVE. Com isso, o presente estudo teve como objetivo descrever os riscos de AVE como complicação neurológica em pacientes acometidos pela COVID-19. A metodologia escolhida foi revisão bibliográfica do tipo revisão integrativa da literatura. Para a realização desse estudo, foram consultadas as bases de dados: MEDLINE, Scholar Google, LILACS, SCIELO e Repositórios. Utilizou-se o DeCS de forma associada: COVID-19, Acidente Vascular Encefálico, Fatores de risco, Neurologia e Coronavírus. Os critérios de inclusão foram: artigos disponibilizados na íntegra, disponíveis nos idiomas português e inglês e publicados entre os anos de 2019 e 2021. Os critérios de exclusão foram: artigos incompletos, cartas ao editor, debates, resenhas, resumos ou artigos publicados em anais de eventos, indisponíveis na íntegra e duplicados. Após a aplicação dos critérios restaram 11 estudos. Sabe-se que a COVID-19 trata-se de um vírus de RNA fita simples nomeado SARS-CoV-2, possuindo afinidade pelo sistema respiratório. Porém, estudos publicados relatam que a COVID-19 também é capaz de atacar o Sistema Nervoso Central podendo ocasionar em outras doenças, como o Acidente Vascular Cerebral, que pode ocorrer através de uma ruptura de um vaso sanguíneo localizado no cérebro, ou pela oclusão do fluxo sanguíneo para o cérebro. A partir disso, torna-se essencial a realização de mais investigações, para que haja uma análise mais profunda acerca de tal temática.
\end{abstract}

Palavras-chave: COVID-19; Acidente vascular encefálico; Fatores de risco; Neurologia; Coronavírus.

\begin{abstract}
In view of the current pandemic scenario caused by COVID-19, it can generate a significant cardiovascular involvement, which is the main risk factor for stroke. Thus, the present study aimed to describe the risks of stroke as a neurological complication in patients affected by COVID-19. The chosen methodology was a bibliographic review of the integrative literature review type. To carry out this study, the following databases were consulted: MEDLINE, Scholar Google, LILACS, SCIELO and Repositories. DeCS was used in an associated way: COVID-19; Brain stroke; Risk factors; Neurology and Coronavirus. Inclusion criteria were: articles available in full, available in Portuguese and English and published between 2019 and 2021. Exclusion criteria were: incomplete articles, letters to the editor, debates, reviews, abstracts or articles published in annals of events, unavailable in full and duplicates. After applying the criteria, 11 studies remained. It is known that COVID-19 is a single-stranded RNA virus named SARS-CoV-2, having an affinity for the respiratory system. However, published studies report that COVID-19 is also able to attack the Central Nervous System, which can cause other diseases, such as stroke, which can occur through a rupture of a blood vessel located in the brain, or by occlusion of the brain. blood flow to the brain. From this, it is essential to carry out further investigations, so that there is a deeper analysis of this theme.
\end{abstract}

Keywords: COVID-19; Brain stroke; Risk factors; Neurology; Coronavirus.

\title{
Resumen
}

Ante el actual escenario pandémico provocado por el COVID-19, puede generar una importante afectación cardiovascular, que es el principal factor de riesgo de ictus. Así, el presente estudio tuvo como objetivo describir los riesgos de ictus como complicación neurológica en pacientes afectados por COVID-19. La metodología elegida fue una revisión bibliográfica del tipo revisión integradora de la literatura. Para la realización de este estudio se consultaron las siguientes bases de datos: MEDLINE, Scholar Google, LILACS, SCIELO y Repositories. El DeCS se utilizó en asociación: COVID-19, accidente cerebrovascular, factores de riesgo, neurología y coronavirus. Los criterios de inclusión fueron: artículos disponibles en su totalidad, disponibles en portugués e inglés y publicados entre 2019 y 2021. Los criterios de exclusión fueron: artículos incompletos, cartas al editor, debates, revisiones, resúmenes o artículos publicados en anales de eventos, no disponibles en su totalidad y duplicados. Después de aplicar los criterios, quedaron 11 estudios. Se sabe que COVID-19 es un virus de ARN monocatenario llamado SARS-CoV-2, 
que tiene afinidad por el sistema respiratorio. Sin embargo, estudios publicados informan que el COVID-19 también es capaz de atacar el Sistema Nervioso Central, lo que puede causar otras enfermedades, como un accidente cerebrovascular, que puede ocurrir por la ruptura de un vaso sanguíneo ubicado en el cerebro o por la oclusión del cerebro. flujo sanguíneo al cerebro. A partir de esto, es fundamental realizar más investigaciones, para que exista un análisis más profundo de este tema.

Palabras clave: COVID-19; Accidente cerebrovascular; Factores de riesgo; Neurología; Coronavirus.

\section{Introdução}

A coronavirus disease 2019 (COVID-19), cujo agente infeccioso foi relatado como um betacoronavírus de RNA envelopado, nomeado de severe acute respiratory syndrome coronavirus 2 (SARS-CoV-2), onde foi relatada inicialmente em dezembro de 2019 na cidade de Wuhan, China. Só houveram confirmações de sua circulação pela Organização Mundial de Saúde (OMS) em janeiro de 2020, e em 11 de março de 2020 foi descrita como uma pandemia pela denominada pela OMS (Kawahara et al., 2020). A doença causa danos cardiorrespiratório, limitações físicas e assim, redução na qualidade de vida (Moura et al., 2021).

O SARS-CoV-2 apresenta algumas características que o torna diferentes dos coronavírus que causaram síndrome respiratória aguda grave (SARS-CoV) zoonótico em 2002 e síndrome respiratória do Oriente Médio (MERS-CoV) em 2012, pois acredita-se que o SARS-CoV-2 tenha 9\% a 96\% similaridade de nucleotídeos com coronavíroses de morcego e seja causado por morcegos, semelhante a outros coronavírus. Como SARS-CoV-1 e MERS, SARS-CoV-2 pode passar de morcegos para um hospedeiro intermediário e posteriormente para humanos (Askin; Tanriverdi; Askin, 2020).

A COVID-19 tem se destacado por ser uma doença infectocontagiosa emergente, já sabe-se que as medidas de prevenção são uma das melhores opções para o combate da disseminação do vírus. Com isso, fronteiras foram fechadas e várias instruções foram estabelecidas, como o isolamento social, orientações a frequente lavagem das mãos, à limpeza e desinfecção de superfícies com mais rigor e o uso obrigatório de máscaras em ambientes públicos (Silva Filho et al., 2021).

Os sintomas da COVID-19, após um período médio de incubação de cinco dias, são febre, tosse seca, fadiga, cefaleia e dispneia. Caso venha a progredir, pode ocorrer um quadro de pneumonia, síndrome do desconforto respiratório, que pode até exigir suporte ventilatório, falência de múltiplos órgãos e morte. Danos potenciais ao sistema nervoso (SN) por coronavírus foram descritos como as causas da síndrome do desconforto respiratório agudo (SDRA) e da síndrome respiratória do Oriente Médio (MERS), com isso algumas manifestações neurológicas foram relataram, como polineuropatia, encefaloite, convulsões epilépticas, acidente vascular cerebral - isquêmico, com achados de autópsia de edema cerebral e vasodilatação menígea (Peña; Quezada; Lemus, 2021).

O Acidente Vascular Encefálico (AVE), também conhecido como Acidente Vascular Cerebral (AVC) é um deficit neurológico súbito que ocorre em uma área cerebral secundário à lesão vascular, além disso a doença pode ter um caráter transitório ou permanente. sabe-se que o AVE é uma das principais causas de morte, mesmo nos países mais desenvolvidos e é também uma queixa prevalente de urgência médica (Santos et al., 2021). Tendo em vista o atual cenário pandêmico ocasionado pela COVID-19 pode gerar um significativo envolvimento cardiovascular, que é o principal fator de risco para o AVE. Com isso, o presente estudo teve como objetivo descrever os riscos de AVE como complicação neurológica em pacientes acometidos pela COVID-19.

\section{Metodologia}

\subsection{Natureza do estudo}

A metodologia escolhida foi revisão bibliográfica do tipo revisão integrativa da literatura (RIL), que apresenta abordagem qualitativa e abrange os estudos com caráter empírico e teórico, identificando ideias já conhecidas sobre um 
assunto especifico em estudos realizados e gerando conhecimentos sobre resultados de distintos estudos (Mendes; Silveira \& Galvão, 2008).

Para estruturar essa revisão seguiram-se 6 etapas metodológicas: identificação do tema e seleção da hipótese ou questão de pesquisa; estabelecimento de critérios para inclusão e exclusão de estudos; busca na literatura; avaliação dos estudos incluídos na revisão integrativa; Interpretação dos resultados; apresentação da revisão ou síntese do conhecimento. RIL se constitui por seis etapas representadas no fluxograma abaixo:

Figura 1 - Fluxograma da revisão.

\section{$1^{\circ}$ - Identificação do tema e Elaboração das perguntas norteadoras}

Quais as principais riscos neurológica em pacientes acometidos pela COVID-19?

Em qual proporção o AVE em pacientes acometidos pela COVID-19?

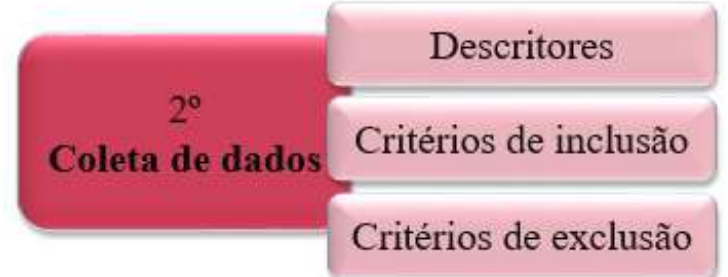

Critérios de exclusão

\section{$4^{\circ}$-Analises críticas das informações a serem extraídas}

\section{$5^{\circ}$ - Interpretação dos resultados}

\section{$6^{\circ}$ - Apresentação da revisão}

\subsection{Estratégia de busca}

Para a realização desse estudo, foram consultadas as bases de dados: Medical Literature Analysis and Retrieval System Online (MEDLINE), Scholar Google, Literatura Latino-americana e do Caribe em Ciências da Saúde (LILACS), Scientific Eletronic Library Online (SCIELO) e Repositórios. Utilizou-se os Descritores em Ciências da Saúde (DeCS) de forma associada: COVID-19, Acidente Vascular Encefálico, Fatores de risco, Neurologia e Coronavírus.

As bases de dados utilizadas foram escolhidas por ser fonte de pesquisa para estudantes e profissionais da área da saúde, por isso, buscou-se identificar as publicações científicas que abordavam o tema deste estudo. Ao final da coleta de dados, foram selecionados os artigos que se adequaram aos objetivos, compondo o corpus de análise.

\subsection{Critérios de inclusão}

Os critérios de inclusão foram: artigos disponibilizados na íntegra, disponíveis nos idiomas português e inglês e publicados entre os anos de 2019 e 2021. 


\subsection{Critérios de exclusão}

Os critérios de exclusão foram: artigos incompletos, cartas ao editor, debates, resenhas, resumos ou artigos publicados em anais de eventos, indisponíveis na íntegra e duplicados.

Para a análise crítica das literaturas foi realizada a análise de Bardin (2011), que consiste em um método que abrange todas as classes de documentos e textos e dividido em três etapas:

1. Pré-análise, nessa etapa ocorreu o acesso as literaturas por meio dos bancos de dados utilizando os descritores e filtros referentes aos critérios de inclusão e exclusão, com intuito de organizar o material a ser utilizado tornando-o operacional;

2. Exploração do material, ocorre a etapa de codificação do material selecionado, nessa fase foi realizado o recorte das unidades de registro, e a categorização em eixos temáticos;

3. No tratamento dos resultados, consistiu na interpretação dos dados em que foram reunidas por características comuns facilitando a apresentação dos resultados através da interpretação de interferência, cuja interpretação consistiu na apresentação dos estudos selecionados e explanação dos eixos temáticos.

\subsection{Análise e interpretação dos dados}

A análise e a interpretação dos dados foram feitas por meio da descrição dos achados. Os dados foram organizados em uma tabela Excel®, que compreendeu as seguintes colunas de sintetização: título do estudo, base de dados, periódico, ano de publicação, contexto/local de estudo, desenho metodológico, resultados e conclusões.

\subsection{Aspectos éticos}

Cabe salientar que os aspectos éticos e os direitos de autoria foram devidamente respeitados, por meio da referenciação dos autores dos trabalhos utilizados. Por se tratar de um estudo de revisão de literatura, não houve necessidade de apreciação por um Comitê de Ética em Pesquisa (CEP).

\section{Resultados e Discussão}

Foram considerados, inicialmente, 205 estudos com a temática proposta; dentre estes, foram excluídos 105 estudos, de acordo com os critérios de exclusão estabelecidos. Após a aplicação dos critérios de inclusão, restaram 11 estudos. Com os estudos incluídos foram feitas revisões críticas e corroborativas sobre cada um deles.

Quadro 1 - Artigos selecionados para discussão da pesquisa (título, autor e ano, objetivo, conclusão).

TITULO

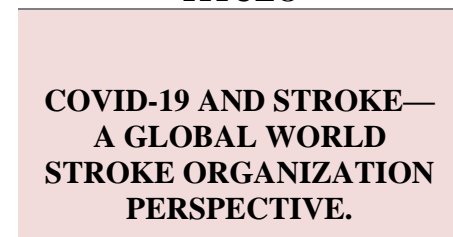

ACIDENTE VASCULAR

CEREBRAL ISQUÊMICO SUBMETIDO A

TROMBÓLISE VENOSA

EM PACIENTE COVID-19

POSITIVO: RELATO DE CASO.

AUTORES / ANO

OBJETIVO

(Markus e Brainin 2020).

(Ribeiro et al., 2021).
Descrever um estudo perspectivo global da Organização Mundial de AVC.

\section{CONCLUSÃO}

A Organização Mundial do AVC tem monitorado o impacto da pandemia globalmente e identificou uma queda inicial acentuada nas apresentações de AVC, bem como um impacto generalizado nos serviços de AVC. A pandemia está mudando a maneira como prestamos atendimento e destacou o enorme potencial da telemedicina no tratamento

Relatar quadro clínico e investigação diagnóstica de AVC isquêmico em paciente infectado por COVID-19, por meio de análise retroativa de prontuário, a fim de se enriquecer o conhecimento sobre o estudo da COVID-19

\section{do AVC}

Os eventos isquêmicos agudos incluem déficit cardiovascular, redução da oxigenação, podendo resultar na Síndrome de Angústia Respiratória

Aguda, e inflamação sistêmica de efeito prótromboembólico. Desse modo, é importante salientar as diversas manifestações sistêmicas da COVID-19. 
COMPLICAÇÕES

CARDIOVASCULARES EM

PACIENTES COM COVID-

19: CONSEQUÊNCIAS DAS

TOXICIDADES VIRAIS E

DA RESPOSTA IMUNE DO HOSPEDEIRO.

COVID-19 E O SISTEMA NERVOSO CENTRAL. como fator importante na

fisiopatologia do AVC.

Revisar o conhecimento atual da biologia do SARS-CoV-2 e os mecanismos potenciais de lesão miocárdica devido a toxicidades virais e respostas imunes do hospedeiro.
O SARS-CoV-2 tornou-se uma ameaça mundial à saúde, com o número de pacientes infectados crescendo rapidamente. Um aumento na incidência de lesão cardíaca foi observada entre aqueles com infecção grave.

Nas infecções por SARS-CoV e MERS foram relatadas manifestações centrais como ansiedade, rebaixamento dos níveis de consciência, alucinações, alterações de humor, agitação.

Entretanto, no caso de SARS-CoV-2, foram patogenia da COVID-19 no sistema nervoso central, com ênfase na teoria da tempestade de citocinas.

observados além dos efeitos centrais já relatados, outras manifestações neurológicas como meningite, encefalite, encefalopatia necrosante aguda (ENA), Síndrome de Giullian-Barré

Relatamos que a incidência combinada de AIS em pacientes com COVID-19 é de 1,2\%, com uma alta taxa de mortalidade. Cima D -dimer, fibrinogénio e a presença de anticorpos antifosfolidos parecem ser proeminente em COVID-19 pacientes com acidente vascular cerebral isquémico concomitante, mas mais estudos mecanísticos são necessários para elucidar o seu papel na patogénese da AIS

Neste estudo de coorte retrospectivo de 2 hospitais acadêmicos da cidade de Nova York, aproximadamente $1,6 \%$ dos adultos com COVID19 que visitaram o departamento de emergência ou foram hospitalizados experimentaram AVC isquêmico, uma taxa maior de AVC em comparação com uma coorte de pacientes com gripe . Estudos adicionais são necessários para confirmar esses achados e investigar possíveis mecanismos trombóticos associados ao COVID-

19.

Pacientes com COVID-19 comumente apresentam manifestações neurológicas. Durante o período epidêmico de COVID-19, ao ver pacientes com manifestações neurológicas, os médicos devem suspeitar de infecção por síndrome respiratória aguda grave por coronavírus 2 como diagnóstico diferencial para evitar diagnóstico tardio ou diagnóstico incorreto e perder a chance de tratar e prevenir transmissão posterior.

No final de 2019, os primeiros casos de COVID-

19 surgiram na cidade de Wuhan, China. Trata-se de um vírus de RNA fita simples nomeado SARS-

CoV-2, possuindo afinidade pelo sistema respiratório. Contudo, artigos publicados recentemente mostraram que o novo coronavírus também é capaz de atacar o Sistema Nervoso Central (SNC) podendo ocasionar em outras doenças, como o Acidente Vascular Cerebral, que pode ocorrer através de uma ruptura de um vaso sanguíneo localizado no cérebro, ou pela oclusão do fluxo sanguíneo para o cérebro.

As informações sobre as manifestações neurológicas relacionadas à SARS-CoV-2 estão disponíveis na forma de alguns grandes estudos e séries de casos, mas as informações gerais estão disponíveis apenas na forma de relatos de casos. As manifestações neurológicas do SARS-CoV-2 são observadas em casos graves de Covid-19. Os relatos de encefalite por SARS-CoV-2 estão na 


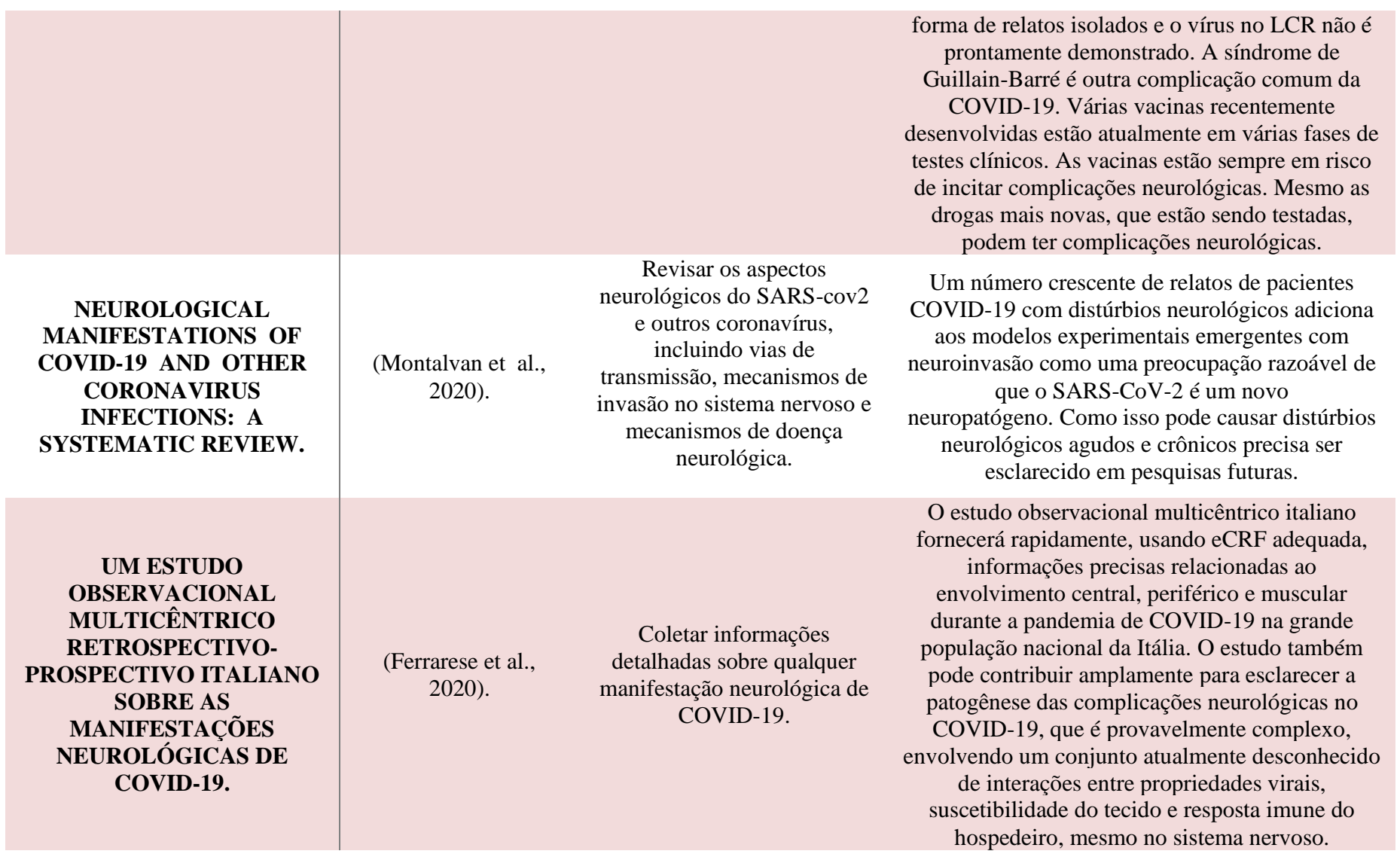

Fonte: Autores (2021).

O novo coronavírus (SARS-CoV-2) além dos riscos pulmonares, também é capaz de causar danos neurológicos. Assim, Markus e Brainin (2020) descreveram que a infecção pela COVID-19 é um fator de risco para o AVE. Já sabe-se que o COVID-19 pode deixar sequelas neurológicas de longo prazo, impactando a saúde pública do país.

Dentre as manifestações dessa infecção, os sintomas neurológicos chamam atenção pela diversidade de apresentação e gravidade. Um estudo observacional retrospectivo feito em Wuhan-China, relatou que 36,4\% dos pacientes apresentação manifestações neurológicas simultâneas a infecção por Sars-CoV-2. Dentre as afecções neurológicas encontradas, anosmia e hiposmia são consideradas sinais característicos precoces do acometimento da COVID-19. Além desses sintomas, também foi relatado o aparecimento de cefaleia, hipogeusia, tontura, confusão, ataxia, epilepsia, dor neuropática, miopatia, encefalopatia necrosante hemorrágica aguda, síndrome de Guillain Barré, meningite e AVE isquêmico (Ribeiro et al., 2021).

No COVID-19, as alterações da pressão arterial é um dos mecanismo sugerido para explicar o risco elevado de manifestações vasculares cerebrais. Pois, a sinalização da enzima conversora de angiotensina 2 (ECA-2) diminui a pressão arterial, além disso, o bloqueio competitivo da enzima conversora de angiotensina 2 pelo vírus SARS-CoV-2 desregula a expressão da ECA- 2, elevando a pressão arterial descontrolada e maior possibilidade de acidentes cerebrovasculares (Zhu et al., 2020).

A infecção pelo SARS-CoV-2 inicia-se por meio da proteína spike (S) que contra-se na superfície do vírus. Essa proteína permite a ligação com um receptor de superfície celular humana chamado de ECA-2. Através de um processo de endocitose e fusão de membranas, o conteúdo da partícula viral é depositado no citoplasma da célula, conforme mostrado na Figura 1. 
Figura 2 - Os mecanismos de invasão do vírus SARS-Cov-2 ao sistema nervoso central (SNC). O vírus possui alta capacidade de penetrar o SNC, sendo por meio do bulbo olfatório, da circulação sanguínea e/ou vias neuronais.

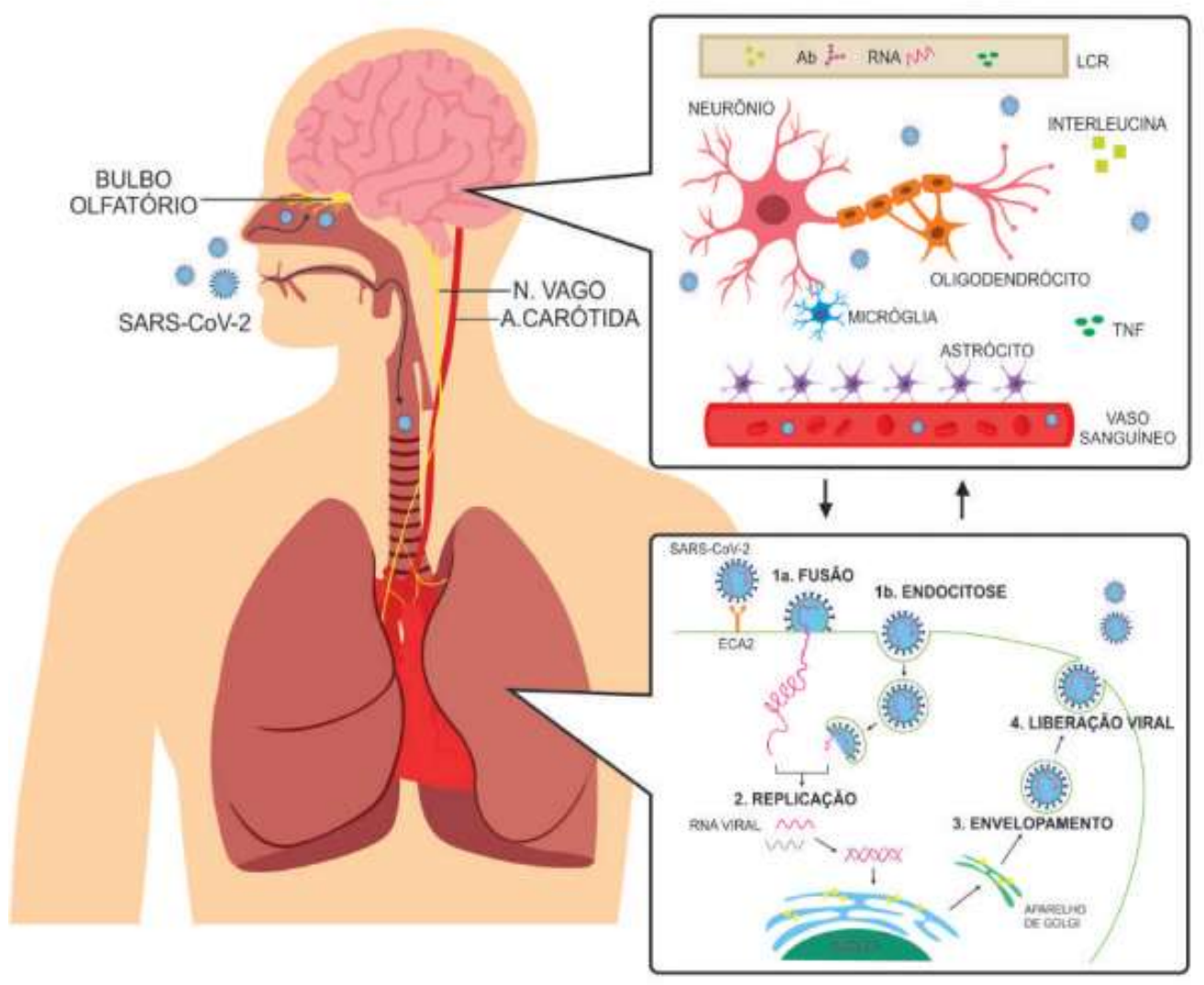

Fonte: Accorsi et al. (2020).

Tan et al. (2020), relatam que a incidência do AVE entre os pacientes com COVID-19 varia entre 0,9\% a 2,7\%. Além disso, o estudo destaca um aumento taxa de mortalidade de 38\% reportada entre os casos. Em um grupo 4466 pacientes com COVID-19, 135 possuíam o AVE, onde 62,3\% eram homens, com média de idade de 63.4 anos, a cerca da comorbidades mais comuns entre os pacientes, se incluíam: Hipertensão arterial sistêmica (64,5\%), Diabetes Mellitus (42,6\%) e Dislipidemias (32\%). A respeito das manifestação de AVE nos pacientes foi considerada de moderada a grave, com elevada prevalência de trombose, embolia e estenose de vasos.

Merkler et al. (2020), descreveram que cerca de 1,5\% dos pacientes infectados pela COVID-19 progrediram com manifestações neurológicas como o AVE do tipo isquêmico, onde ocorre quando um vaso sanguíneo cerebral importante é obstruído pela formação de um trombo em seu interior, muitas vezes sobre uma artéria comprometida pela aterosclerose, ou pela migração de um coágulo sanguíneo formado no interior do coração.

Um dos primeiros estudos publicados foi sobre 99 pacientes averiguado nos primeiros 20 dias do mês de janeiro de 2020 na cidade de Wuhan e, foi concluído que 40\% dos pacientes com COVID-19 já apresentavam uma doença cardiovascular ou cerebrovascular (Mao et al., 2020). Dentre eles, apenas os quatro primeiros analisaram as complicações ou as causas de morte e, entre esses quatro, apenas um analisou as manifestações neurológicas (Godoy; Yaman, 2020).

Os pacientes mais graves em sua maioria são idosos (> 60 anos), que no geral apresentam comorbidades. Porém, há um número crescente de relatos de jovens COVID-19 positivos sem comorbidades que sofreram AVE. Um estudo demonstrou que o AVE agudo é o achado de neuroimagem mais comum relacionado à COVID-19, e é considerado marcador de mau prognóstico (Garg, 2020). 
O estudo de Montalvan et al. (2020), demonstra que os pacientes com históricos ou fatores de risco vasculares, possuem maiores chances desenvolverem o AVE com a presença de hipotensão, insuficiência cardíaca, e choque, que contribuem na hipoperfusão, mecanismos embólicos do AVE e oclusão de grandes vasos. No estudo observacional realizado na China com 191 pacientes, pode-se observar que 23\% sofreram complicações com insuficiência cardíaca, 20\% com choque séptico, $19 \%$ com coagulopatia e $17 \%$ com lesão cardíaca aguda, além disso foi relatado que essas condições acarretam no quadro de AVE.

A partir disso, pode-se verificar que o SARS-CoV-2, pode atingir o SN, músculo esquelético e trato respiratório, isso ocorre devido todos os sistemas fazerem parte dos mesmos receptores ACE-2 presentes no sistema respiratório. À medida que o número de pacientes com COVID-19 aumenta em todo o mundo, as manifestações neurológicas têm sido rotineiramente relatadas. Por todas essas razões, o monitoramento próximo de qualquer possível manifestação neurológica no início, durante ou após COVID-19, é obrigatório para a comunidade neurológica (Ferrarese et al., 2020).

\section{Considerações Finais}

Sabe-se que a COVID-19 trata-se de um vírus de RNA fita simples nomeado SARS-CoV-2, possuindo afinidade pelo sistema respiratório. Porém, estudos publicados relatam que a COVID-19 também é capaz de atacar o Sistema Nervoso Central (SNC) podendo ocasionar em outras doenças, como o Acidente Vascular Cerebral, que pode ocorrer através de uma ruptura de um vaso sanguíneo localizado no cérebro, ou pela oclusão do fluxo sanguíneo para o cérebro.

Visto isso, faz-se necessário pesquisas futuras sobre a relação entre a COVID-19 e o AVE, observou-se que os pacientes acometidos, obtiveram maior gravidade e prognósticos desfavoráveis na afecção. A partir disso, torna-se essencial a realização de mais investigações, para que haja uma análise mais profunda acerca de tal temática. Portanto, com a realização de futuros trabalhos pela comunidade científica, os danos neurológicos serão mais averiguados e o conhecimento será gerado para um melhor manejo clínico aos pacientes acometidos pelas afecções neurológicas com COVID-19.

\section{Referências}

Accorsi, D. X., dos Santos, I. A. M., Accorsi, J. X., Bohac, S., \& Chin, C. M. (2020). COVID-19 e o Sistema Nervoso Central. Ulakes Journal of Medicine, 1.

Askin, L., Tanrıverdi, O., \& Askin, H. S. (2020). O efeito da doença de coronavírus 2019 nas doenças cardiovasculares. Arquivos brasileiros de cardiologia, 114, 817-822.

Bardin, L. (2011). Analise de Conteúdo. Ed. Revista Ampliada.

Ferrarese, C., Silani, V., Priori, A., Galimberti, S., Agostoni, E., Monaco, S., \& Sociedade Italiana de Neurologia (SIN). (2020). Um estudo observacional multicêntrico retrospectivo-prospectivo italiano sobre as manifestações neurológicas de COVID-19 (NEUROCOVID). Neurological Sciences, 41 . $1355-1359$.

Garg, R. K. (2020). Spectrum of neurological manifestations in Covid-19: a review. Neurology India, 68(3), 560.

Godoy, M. D. S., \& Yamane, F. D. O. (2020). Acidente Vascular Cerebral Na Pandemia Por Covid-19.

Kawahara, L. T., Costa, I. B. S. D. S., Barros, C. C. S., Almeida, G. C. D., Bittar, C. S., Rizk, S. I., \& Hoff, P. M. (2020). Câncer e Doenças Cardiovasculares na Pandemia de COVID-19. Arquivos Brasileiros de Cardiologia, 115, 547-557.

Mao, L., Jin, H., Wang, M., Hu, Y., Chen, S., He, Q., \& Hu, B. (2020). Neurologic manifestations of hospitalized patients with coronavirus disease 2019 in Wuhan, China. JAMA neurology, 77(6), 683-690.

Markus, H. S., \& Brainin, M. (2020). COVID-19 and stroke-A global World Stroke Organization perspective. International journal of stroke, 15(4), 361364.

Mendes, K. D. S., Silveira, R. C. D. C. P., \& Galvão, C. M. (2008). Revisão integrativa: método de pesquisa para a incorporação de evidências na saúde e na enfermagem. Texto \& contexto-enfermagem, 17(4), 758-764.

Merkler, A. E., Parikh, N. S., Mir, S., Gupta, A., Kamel, H., Lin, E., \& Navi, B. B. (2020). Risk of ischemic stroke in patients with coronavirus disease 2019 (COVID-19) vs patients with influenza. JAMA neurology, 77(11), 1366-1372.

Montalvan, V., Lee, J., Bueso, T., De Toledo, J., \& Rivas, K. (2020). Neurological manifestations of COVID-19 and other coronavirus infections: A 
systematic review. Clinical Neurology and Neurosurgery, 194, 105921.

Moura, J. M., Silva, L. M., de Souza, R. F., \& Ramos, D. V. B. (2021). Indicação e uso de azitromicina no tratamento do COVID-19: revisão da literatura. Brazilian Journal of Development, 7(6), 56547-56556.

Peña, S. L., Quezada, M. E. B., \& Lemus, V. S. (2021). Manifestaciones neurológicas y COVID-19. Alerta, Revista científica del Instituto Nacional de Salud, 4(2), 69-80.

Ribeiro, L. M., Balestrero, J. G. P., Borges, S. D. O. R. F., de Oliveira, N. C., Pezzin, F. S., de Freitas, P. M., \& Júnior, J. A. F. (2021). Acidente vascular cerebral isquêmico submetido a trombólise venosa em paciente Covid-19 positivo: relato de caso. Brazilian Journal of Health Review, 4(2), 7319-7332.

Santos, D., Marques, G., Almeida, L., Holanda, A., Guedes, I., \& de Oliveira Dutra, J. C. (2021). AVC COMO COMPLICAÇÃO DA INFECÇÃO POR COVID-19. Estudos Avançados Sobre Saúde E Natureza, 1.

Silva Filho, P. S. P., de Sousa Figueiredo, B. L., Kirchesch, C. L., Amaral, S. M., dos Santos, L. D. L., Ferreira, J. P. N., \& de Carvalho Oliveira, C. P. (2021). Pneumonia ocasionada pela COVID-19 e a importância do diagnóstico como benefício para o tratamento. Research, Society and Development, 10(5), e14710514600-e14710514600.

Tan, Y.-K., Goh, C., Leow, A. S. T., Tambyah, P. A., Ang, A., Yap, E.-S., Tu, T.-M., Sharma, V. K., Yeo,L. L. L., Chan, B.P. L., \& Tan, B. Y. Q. (2020). COVID-19 and ischemic stroke: a systematic review and meta-summary of the literature. Journal of Thrombosis and Thrombolysis, 50(3), 587-595.

Zhu, H., Rhee, JW, Cheng, P., Waliany, S., Chang, A., Witteles, RM, \& Wu, SM (2020). Complicações cardiovasculares em pacientes com COVID-19: consequências das toxicidades virais e da resposta imune do hospedeiro. Relatórios atuais de cardiologia , 22 (5), 1-9. 